Revista Electrónica Complutense de Investigación en Educación Musical ISSN-e: 1698-7454

hhttp://dx.doi.org/10.5209/RECIEM.53381

\title{
¿Cómo enseñan nuestros profesores de instrumento musical? Situación de la enseñanza de estrategias de práctica efectiva en seis escuelas de música en México ${ }^{1}$
}

\author{
Raúl W. Capistrán Gracia²
}

Recibido: 13 de septiembre de 2016 / Aceptado: 07 de julio de 2017

Resumen. Este artículo presenta los resultados de un estudio exploratorio que tuvo como objetivo determinar el nivel de enseñanza de estrategias de práctica efectiva por parte de 84 profesores de instrumento en seis departamentos universitarios de música en México. A través de la técnica de la encuesta, los profesores respondieron un cuestionario que incluyó preguntas contextuales y preguntas con escalas tipo Likert y opción múltiple para determinar su nivel de compromiso para enseñar estrategias de practica efectiva, la manera de monitorizar la práctica de sus estudiantes, el grado de satisfacción con su desempeño, su opinión respecto a los recursos que podrían mejorar el proceso de enseñanza y aprendizaje instrumental, la manera como se mantienen actualizados y su filosofía de enseñanza. Los datos fueron capturados y analizados con la ayuda del programa de computación Statistics Package for the Social Sciences IBM SPSS 2.0. Los resultados sugieren que los profesores encuestados poseen un alto nivel de compromiso; sin embargo, se detectaron algunos aspectos mejorables, tales como deficiencias en la enseñanza de estrategias relacionadas con el control de ejecución y la autoevaluación, falta de monitorización efectiva de la práctica de sus estudiantes, así como falta de interés en fundamentar su práctica docente en resultados de investigación. Adicionalmente, los resultados indican que las instituciones se preocupan poco por promover la impartición de cursos de pedagogía del instrumento. El investigador propone algunas recomendaciones en las secciones de discusión y conclusiones.

Palabras clave: práctica efectiva; autorregulación; metacognición; monitorización de la práctica; formación profesional.

\section{[en] How do our professors teach playing musical instruments? Situation of effective practice strategies teaching in six music schools in Mexico}

\begin{abstract}
This article presents the results of an exploratory study that aimed to determine the level of teaching of effective practice strategies by 84 instrument teachers in six university music schools in Mexico. Through the survey technique, teachers answered a questionnaire that included contextual questions as well as questions with Likert scales and multiple choice answers to identify some aspects related to their teaching, such as their level of commitment to teach effective practice strategies, the way they monitored the practice of their students, the level of satisfaction with their performance, their opinion concerning what resources would improve the teaching-learning process, the way they kept updated, as well as their teaching philosophy. The data were captured and analyzed with the help of the software Statistics Package for the Social Sciences, IBM SPSS 2.0. The results suggested surveyed teachers have a high level of commitment with their profession. However, some weaknesses were
\end{abstract}

\footnotetext{
Programa para el Desarrollo Profesional Docente, para el Tipo Superior (PRODEP)

2 Universidad Autónoma de Aguascalientes

E-mail rwcapistran@correo.uaa.mx
} 
identified such as: deficiencies in teaching strategies in the dimension of control of execution and self-evaluation of practice, lack of effective monitoring of their students' practice, as well as lack of interest to support their practicum on research results. In addition, results suggested institutions are not concern on promoting courses on pedagogy of the instrument. Some recommendations are provided in the discussion and conclusion sections.

Keywords: Effective practice; self-regulation; metacognition; monitoring of practice; professional formation.

Sumario. 1. Introducción. 2. Marco teórico. 3. Objetivos. 4. Método: Participantes; materiales; procedimiento. 5. Resultados y discusión: Dimensión de preparación de la práctica; dimensión de control de ejecución; dimensión de auto-redireccionamiento; resumen estadístico del uso de estrategias. 6 . Conclusiones. 7. Referencias bibliográficas

Como citar: Capistrán Gracia, R. W. (2017). ¿Cómo enseñan nuestros profesores de instrumento musical? Situación de la enseñanza de estrategias de práctica efectiva en seis escuelas de música en México. Revista Electrónica Complutense de Investigación en Educación Musical, 14, 213-237.

\section{Introducción}

Como es sabido, los estudiantes de música realizan la mayor parte del aprendizaje de sus obras en soledad (Gaunt, 2008; Jorgensen, 2000; Sloboda, Davidson, Howe y Moore, 1996) donde deben decidir cómo organizar su práctica y qué estrategias utilizar para aprender sus obras (Byo y Cassidy, 2008; Oare, 2012). Por lo anterior, la tarea más importante del profesor de instrumento, en los primeros años de formación profesional de sus estudiantes, consiste en enseñarles estrategias efectivas en su aprendizaje, estableciendo rutinas de estudio productivas y saludables. De esta forma ellos podrán obtener mejores resultados de forma progresiva (Pearce, 1992). Gradualmente, el profesor deberá enseñar y promover el desarrollo de habilidades metacognitivas en sus estudiantes, de tal manera que éstos aprendan a aprender y lleguen a ser independientes (Hallam, 2001a; Jorgensen, 2000).

Esta labor, tiene una importancia relevante, ya que los estudiantes con poca experiencia suelen no ser capaces de diseñar un régimen de práctica efectiva para aprender sus obras (Barry, 1990) y pueden tardar años en tener conciencia de la necesidad de aprender estrategias de práctica efectiva (Bugos y High, 2009).

Desgraciadamente, en las lecciones de instrumento, muchos profesores se centran principalmente en los resultados finales de interpretación, tal como lo describen algunos autores en sus investigaciones (Gaunt, 2008; Koopman, Smith, de Vugt, Deneer y den Ouden, 2007; Kostka, 2002; Young, Burwell y Pickup, 2003). Por lo que se limitan generalmente a abordar aspectos técnicos y expresivos de las piezas que están tocando los estudiantes, sin involucrarse en el proceso de aprendizaje del alumno (Duke, Simmons y Cash, 2009; Gaunt, 2008; Jorgensen, 2000; Koopman et ál., 2007, Laukka, 2004).

Por lo anterior se puede argumentar que, promover el uso de estrategias de práctica efectiva por parte de los profesores es vital durante la formación del músico profesional. De modo que el presente artículo se centra en presentar los resultados de un estudio exploratorio, que tuvo como objetivos determinar el nivel de enseñan- 
za de estrategias de práctica efectiva por parte de 84 profesores de instrumento de seis departamentos universitarios de música en México, así como identificar algunos aspectos relacionados con su labor docente, tales como la manera como monitorizan la práctica de sus estudiantes, el nivel de satisfacción con su desempeño, la manera como se mantienen actualizados y su filosofía de enseñanza del instrumento. Esto con el propósito de detectar los aspectos mejorables en su práctica docente y proponer medidas de mejora.

\section{Marco Teórico}

Siguiendo a Zimmerman $(1998,2000)$ la práctica efectiva se podría definir como una actividad cíclica organizada en tres grandes dimensiones: preparación (métodos que preceden los esfuerzos de aprendizaje), control de ejecución (métodos que ocurren durante los esfuerzos de aprendizaje) y auto re-direccionamiento (métodos que ocurren después de los esfuerzos de aprendizaje). Ese auto re-direccionamiento se refiere, específicamente, a una auto-evaluación de la práctica. Así, ejerce gran influencia en la preparación respecto a los subsiguientes esfuerzos de aprendizaje, con lo que se completa el círculo auto-regulatorio e impulsa su re-inicio.

Cada fase o dimensión del ciclo auto-regulatorio (preparación, control de ejecución y auto re-direccionamiento) involucra el uso de estrategias de práctica efectiva específicas. Sin embargo, no son estrategias fijas, sino que deberán ser seleccionadas por el estudiante de acuerdo a un contexto o situación (Zimmerman, 1998). Muchas de ellas han sido utilizadas desde siempre. Sin embargo, su estudio, desde la perspectiva de la investigación empírica, ha arrojado luz sobre su valor y su aplicación metodológica y ha contribuido a fundamentar o no fundamentar su uso, con lo que se derrumban los prejuicios que sobre algunas de ellas existían.

Así, en lo que se refiere a estrategias de preparación de la práctica, se sabe que establecer un horario específico para practicar, constituye una manera de autorregularse característica de estudiantes talentosos (Hallam, 2001b). De modo que esto determina que la práctica sea más efectiva cuando está dispuesta de manera lógica y secuencialmente estructurada (Barry, 1990; Miksza, 2011); así mismo, establecer objetivos claros y realistas ayuda a mantener la concentración, favorece el crecimiento musical (Byo y Cassidy, 2008) estimula el esfuerzo, incrementa la persistencia y favorece el desarrollo de estrategias (Lathman, 2004).

Del mismo modo, el uso de diarios y bitácoras promueve la reflexión, fomenta la meta-cognición (Cremaschi, 2012) además de favorecer la concentración (Kim, 2008). En ese mismo rubro, realizar anotaciones en la partitura se encuentra entre las tareas que llevan a cabo los músicos avanzados (Teixeira-Dos Santos y Hentschke, 2011).

Otros estudios empíricos indican que, planificar la interpretación musical de una obra sobre la base del análisis musical antes de practicarla, constituye una característica de músicos profesionales con una amplia preparación (Hallam, 1995) y que llevar a cabo un análisis formal y armónico de las obras de estudio, contribuye a una comprensión más profunda y favorece la memorización (Herrera y Cremades, 2014).

Por lo que respecta a estrategias involucradas en la fase de control de ejecución, se ha comprobado que practicar una sección a la vez es una estrategia propia de es- 
tudiantes con altos niveles de autorregulación (Miklaszewski, 1989; Nielsen, 2001). Del mismo modo, aislar los pasajes difíciles para practicarlos por separado es una de las estrategias más efectivas, no sólo para vencer dificultades técnico-musicales, sino también para memorizar la música (Maynard, 2006).

En cuanto a practicar las piezas de principio a fin sin detenerse, Swanson y Law (1993) consideran que la estrategia de alternar la práctica de secciones con interpretaciones completas de la obra, es necesaria para lograr un aprendizaje holístico de las mismas. Practicar de manera "piramidal" (estrategia de tipo aditivo en donde se aprende una sección o frase por vez y se van uniendo hasta aprender un todo), es propio de estudiantes con alto nivel de auto-regulación (Nielsen, 2001). Lo mismo podría decirse de otras variantes de esta estrategia, tales como practicar la última frase, luego la penúltima y luego la antepenúltima hasta aprender una sección completa (Berr, 2010), o practicar partiendo de una sección del centro e ir añadiendo frases antes y después.

Asimismo, practicar lentamente y practicar con metrónomo son estrategias relacionadas con el logro y el crecimiento musical (Duke, et ál., 2009; Hallam, Rinta, Varvarigou, Creech, Papageorgi, Gomes y Lanipekun, 2012; Miksza, 2007 y 2011; Nielsen, 2001).

Por otro lado, los estudios de Shockley (1997) indican que, crear mapas mentales es una estrategia de alto nivel metacognitivo, pues requiere que el estudiante utilice todos sus conocimientos teóricos a fin de establecer conexiones o relaciones que le permitan registrar mentalmente el evento teórico-sonoro. En ese mismo sentido, un número importante de estudios indican que la ejercitación mental es una estrategia importante en el crecimiento y el logro musical y constituye una de las formas más importantes de promover la meta-cognición (Coffman, 1990; Johnson, 2011; Reybrouck, 2009; Theiler y Lippman, 1995) y prepararse para una presentación (Gregg y Clark, 2007).

Barry y McArthur (1994), Brooks (1995) y Holmes (2005) recomiendan combinar la práctica real y la ejercitación mental, ya que practicar de esa manera promueve habilidades metacognitivas de orden superior. Adicionalmente, la ejercitación mental ayuda a mantener un tiempo estable (Johnson, 2011), contribuye a una memorización más efectiva (Holmes, 2005), limita las distracciones, mantiene la agudeza mental, ayuda a corregir errores, favorece una ejecución más segura (Gregg, Clark y Hall, 2008) y promueve el logro de mejores resultados (McPherson y McCormick, 1999).

Finalmente, darse instrucciones a uno mismo (auto-guiarse) es una estrategia que promueve procesos meta-cognitivos de nivel superior y parece ser una característica de estudiantes altamente autorregulados y exitosos (Byo y Cassidy, 2008; Miksza, 2006; Nielsen, 2001; Williamon y Valentine, 2000; Zimmerman, 2008).

En cuanto a estrategias involucradas en la fase de re-direccionamiento se puede incluir la grabación en video y la grabación en audio como medios de autoevaluación. De acuerdo a diversos estudios empíricos, estas estrategias de práctica efectiva ayudan al estudiante a determinar si ha mejorado y a seleccionar la estrategia más adecuada para vencer los retos técnico-musicales (McPherson y McCormick, 1999; McPherson y Zimmerman, 2002; Nielsen, 1999; Rosenthal, Durairaj y Magann, 2009). Al mismo tiempo, agudiza el criterio de autoevaluación del estudiante (Daniel, 2001; Silveira y Gavin, 2015) y promueve la reflexión sobre el proceso de aprendizaje (Boucher, Dube y Creech, 2017). 
Para terminar, la ejecución instrumental frente a un compañero (evaluación de pares) contribuye al logro de mejores resultados, desarrolla la destreza para resolver problemas y facilita el entendimiento entre estudiantes (Sheldon, 2005).

\section{Objetivos}

1) Identificar las estrategias de práctica efectiva y autorregulación que los maestros enseñan a sus estudiantes cuando éstos se preparan para una actuación y la medida en que cubren las dimensiones de preparación, control de ejecución y auto re-direccionamiento definidas por Zimmerman $(1998,2000)$.

2) Determinar el grado en que los maestros monitorizan la práctica de sus estudiantes.

3) Determinar la manera como los maestros monitorizan la práctica de sus estudiantes.

4) Determinar el nivel de satisfacción de los maestros respecto a los resultados que los estudiantes obtienen en sus sesiones de práctica y en sus presentaciones.

5) Conocer la opinión de los maestros respecto al tipo de recursos que podrían contribuir para que los estudiantes mejoren su desempeño como ejecutantes.

6) Determinar la frecuencia con que los maestros reciben cursos sobre pedagogía del instrumento que aborden el tema de las estrategias de práctica.

7) Caracterizar la filosofía de los maestros en cuanto a la enseñanza instrumental.

\section{Método}

\subsection{Participantes}

En el estudio participaron 84 profesores de pregrado de diversos instrumentos (es decir, profesores que enseñan cómo tocar un instrumento, no profesores de materias teóricas) de seis departamentos de música pertenecientes a universidades públicas de la Región Centro Occidente de la Asociación Nacional de Universidades e Instituciones de Educación Superior (ANUIES) ${ }^{3}$. La muestra tomada fue de oportunidad o conveniencia (Creswell, 2001). Sin embargo, un cálculo estadístico posterior reveló que tiene un $7 \%$ de margen de error y un $93 \%$ de nivel de confianza. Las escuelas participantes se consignan en la siguiente tabla:

La Asociación Nacional de Universidad e Instituciones de Educación Superior en México (ANUIES) ha organizado al país en seis regiones. La Región Centro Occidente incluye seis estados. La muestra incluye seis departamentos de música de seis universidades públicas, uno por cada estado de esa región. 
Tabla 1. Universidades participantes y número de profesores participantes.

\begin{tabular}{lll}
\hline Universidad & $\begin{array}{l}\text { Número de profesores que } \\
\text { imparten clase de instrumen- } \\
\text { to }\end{array}$ & $\begin{array}{l}\text { No. de Profesor de Instru- } \\
\text { mento encuestados }\end{array}$ \\
\hline $\begin{array}{l}\text { Universidad Autónoma } \\
\text { Aguascalientes }\end{array}$ & 19 & 13 \\
$\begin{array}{l}\text { Universidad de Colima } \\
\text { Universidad Autónoma de }\end{array}$ & 14 & 10 \\
$\begin{array}{l}\text { Nayarit } \\
\text { Universidad de Guadalajara }\end{array}$ & 32 & 11 \\
$\begin{array}{l}\text { Universidad de Guanajuato } \\
\text { Universidad Michoacana }\end{array}$ & 34 & 19 \\
de San Nicolás de Hidalgo & 35 & 16 \\
Total & 145 & 15 \\
\hline
\end{tabular}

Las edades de los profesores oscilaron entre los 27 y los 76 años, con un promedio de 45.32 años y una mediana de 46 años. De los participantes, 52 fueron hombre y 26 fueron mujeres (hubo seis participantes que no proporcionaron su género).

Por lo que respecta al nivel académico, un $53.3 \%$ de los profesores cuenta con licenciatura, un $26.7 \%$ cuenta con maestría mientras que un pequeño $18.7 \%$ afirma tener un doctorado.

Tabla 2. Nivel académico de los profesores.

\begin{tabular}{|l|l|l|l|}
\hline & Frecuencia & Porcentaje & Porcentaje Valido \\
\hline Diplomado & 1 & 1.2 & 1.3 \\
\hline Licenciatura & 40 & 47.6 & 53.3 \\
\hline Maestría & 20 & 23.8 & 26.7 \\
\hline Doctorado & 14 & 16.7 & 18.7 \\
\hline Total & 75 & 89.3 & 100.0 \\
\hline Valores perdidos & 9 & 10.7 & \\
\hline Total & 84 & 100.0 & \\
\hline
\end{tabular}

\subsection{Materiales}

El estudio fue de tipo exploratorio y de corte cuantitativo. La escala para determinar el grado de enseñanza de estrategias de práctica efectiva y la medida en que cubren las dimensiones de preparación, control de ejecución y auto re-direccionamiento definidas por Zimmerman $(1998,2000)$ consistió de 30 ítems con escalas tipo Likert, con una posibilidad de respuesta de 1 (nunca) a 5 (siempre). A través de una revisión bibliográfica a profundidad, se detectaron y seleccionaron estrategias cuyo valor metodológico estuviera fundamentado por estudios empíricos y que correspondieran 
a cada una de las dimensiones de práctica efectiva, de acuerdo a la descripción y ejemplos que Zimmerman proporciona $(1998,2000)$. Cada una de las estrategias de práctica efectiva seleccionadas fue abordada en el marco teórico y está representada por cada uno de los ítems del cuestionario. Para validar la escala se utilizó la técnica de juicio de expertos, en la que participaron tres, uno del Institute of Education de la University College London y dos del Departamento de Música de la Universidad donde labora el investigador. La confiabilidad de esta escala fue buena, con un coeficiente alfa de Cronbach de 0.784 . Tanto la escala de 30 ítems, como el instrumento completo pasaron por numerosas pruebas piloto. Las observaciones de los profesores voluntarios, aunque mínimas, fueron atendidas hasta que quedaron satisfechos.

Adicionalmente, el cuestionario incluyó preguntas contextuales y preguntas con escalas tipo Likert y opción múltiple para identificar, desde la perspectiva de los profesores, algunos aspectos relacionados con su labor docente, tales como el grado o nivel de compromiso de los profesores para enseñar estrategias de practica efectiva, la manera de monitorizar la práctica de sus estudiantes, el nivel de satisfacción con el desempeño de sus estudiantes, su opinión respecto a los recursos que podrían mejorar el proceso de enseñanza y aprendizaje instrumental, la manera como se mantienen actualizados y su filosofía de enseñanza.

\subsection{Procedimiento}

Los cuestionarios fueron administrados por el propio investigador. Las escuelas fueron visitadas durante el mes de octubre y principios de noviembre de 2015. Los profesores fueron abordados durante sus descansos o cuando ingresaban al departamento de música para laborar. El llenado de los cuestionarios fue voluntario y anónimo. Los datos fueron capturados y analizados con la ayuda del programa de computación Statistics Package for the Social Sciences IBM SPSS 2.0.

\section{Resultados y Discusión}

Después de la sección de datos generales (género, nivel académico, etc.) el cuestionario incluyó preguntas relacionadas con la enseñanza y monitorización de la práctica. La primera pregunta: “¿Enseña usted a sus estudiantes estrategias de práctica para aprender sus obras en preparación para una actuación?" recibió un alto "siempre" $(72.60 \%)$ como respuesta principal y un $23.80 \%$ para "frecuentemente".

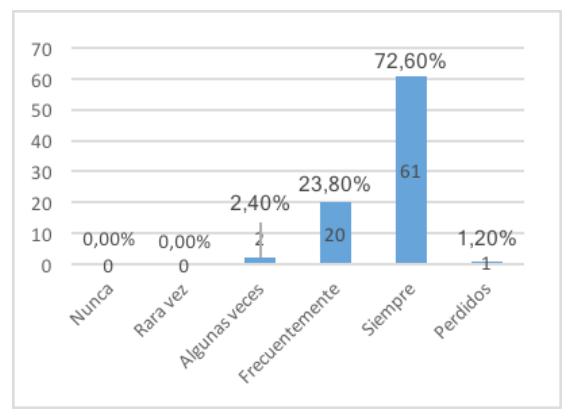

Figura 1. Profesor que enseñan estrategias de práctica a sus estudiantes. 
Como se mencionó anteriormente, la mayor parte del tiempo, los estudiantes practican solos, sin que los profesores tengan idea de lo que pasa en el salón de práctica (Gaunt, 2008; Jorgensen, 2000; Miksza, 2006; Sloboda, et ál., 1996). Muchas veces la práctica no es la adecuada y el estudiante no tiene manera de recibir retroalimentación hasta una semana después, cuando vuelve a la lección de instrumento. Una propuesta del investigador, es que el profesor debe implementar maneras de monitorizar la práctica de sus estudiantes. Podría ser que, a la mitad de la semana, el estudiante vaya con el profesor para mostrarle cómo está practicando o que, en la lección de instrumento, cuando el estudiante regresa a presentar sus avances, el profesor se tome tiempo para verificar que el estudiante esté utilizando estrategias de práctica efectivas. Podría ser también que el estudiante se auto grabe en video o en audio y muestre al profesor cómo practicó un pasaje, que lleve un registro de las maneras como ha practicado o mejor aún, que elabore un portafolio de evidencias sobre su práctica.

El profesor podría implementar acciones más ingeniosas, como pedirle al estudiante que vaya directamente a los pasajes difíciles y le muestre cómo los está practicando. Por todo lo anterior se incluyó la pregunta número 2 “Monitoriza usted la manera como practican sus estudiantes?" Las respuestas no fueron tan abrumadoras como en el ítem anterior; un $41.70 \%$ afirmó hacerlo siempre, un $29.80 \%$ aseguró hacerlo frecuentemente mientras que un $23.80 \%$ afirmó hacerlo algunas veces.

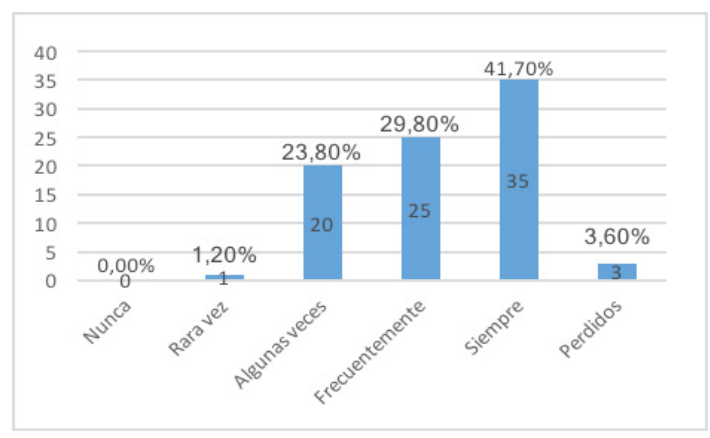

Figura 2. Profesor que monitorizan la práctica de sus estudiantes.

Las respuestas proporcionadas a la pregunta 3 “Cómo monitoriza usted la práctica de sus estudiantes?" Sugieren que en realidad no existe una monitorización efectiva. Solo un $21 \%$ de los encuestados afirmó pedir a sus estudiantes que practicaran enfrente de ellos para valorar la calidad de la práctica. Un alto 37\% afirmó cerciorarse de que los estudiantes sabían lo que tenían que hacer antes de terminar la lección. Sin embargo, muchos estudiantes no siguen las indicaciones de los profesores cuando practican por su cuenta, lo que Flavell, Beach y Chinsky (1966) llaman "deficiencia de producción".

Un 29\% afirmó evaluar la práctica por los resultados que los estudiantes obtienen durante la lección. Sin embargo, los resultados presentados durante las lecciones, pueden ser engañosos, ya que el estudiante, al no estar sometido al estrés de una ejecución pública, puede estar confiando más en su intuición y en su memoria kinestésica que en un conocimiento sólido y bien fundamentado producto de la práctica efectiva (Bernstein, 1981; Westney, 2003). Finalmente, un 8\% de los profesores consideró que los estudiantes deben responsabilizarse de su propio aprendizaje. Sin embargo, esta parte 
tan importante de la formación profesional del músico, no es sólo responsabilidad del estudiante sino de toda la comunidad académica (Jorgensen, 2000).

Tabla 3. Manera como los profesores monitorizan la práctica de sus estudiantes.

\begin{tabular}{lll}
\hline a) Por los resultados que ellos obtienen durante la lección & 40 & $29 \%$ \\
b) Les pido que practiquen delante de mi & 29 & $21 \%$ \\
$\begin{array}{l}\text { c) Antes de terminar la lección me cercioro de que saben } \\
\text { cómo van a practicar }\end{array}$ & 50 & $37 \%$ \\
$\begin{array}{ll}\text { d) Considero que los estudiantes deben responsabilizarse de } \\
\text { su propio aprendizaje. Yo solamente los oriento }\end{array}$ & \\
e) Otro & 11 & $8 \%$ \\
$\quad$ Total & 6 & $4 \%$ \\
\hline
\end{tabular}

Al entrecruzar los datos se encontró que los profesores que piden a sus estudiantes que practiquen delante de ellos para corroborar la efectividad de la práctica, son en su mayoría, aquellos que tienen nivel de posgrado.

Tabla 4. Tabla de Contingencia: Monitorización de la práctica y nivel académico.

\begin{tabular}{l|ll}
\hline \multirow{5}{*}{ Diplomado } & Frecuencia & 0 de 1 \\
& $\%$ dentro del Nivel Académico & $0.0 \%$ \\
& $\%$ del Total & $0.0 \%$ \\
Licenciatura & Frecuencia & 8 de 40 \\
& $\%$ dentro del Nivel Académico & $20.0 \%$ \\
& $\%$ del Total & $10.7 \%$ \\
\hline \multirow{3}{*}{ Maestría } & Frecuencia & 10 de 20 \\
& $\%$ dentro del Nivel Académico & $50.0 \%$ \\
Doctorado & $\%$ del Total & $13.3 \%$ \\
\hline Perdidos & Frecuencia & 8 de 14 \\
\hline
\end{tabular}

La cuarta pregunta o sección de la encuesta, estuvo representada por la escala de 30 ítems con escalas tipo Likert para determinar el nivel de enseñanza e implementación de estrategias de práctica efectiva por parte de los profesores y la medida en que cubren las tres dimensiones definidas por Zimmerman (1998, 2000): preparación, control de ejecución y auto re-direccionamiento.

\subsection{Dimensión de preparación de la práctica}

Los ítems IV-1 al IV-13 y IV-27 (catorce ítems) tuvieron como objetivo determinar el nivel de enseñanza de estrategias de práctica efectiva para llevar a cabo una prepa- 
ración efectiva de la práctica instrumental. El alfa de Cronbach para esta dimensión fue de 0.791 .

Tabla 5. Lista de ítems que conformaron la dimensión de "preparación de la práctica".

\begin{tabular}{ll}
\hline & \multicolumn{1}{c}{ Ítems } \\
\hline IV 1 & Le pido a mis estudiantes que establezcan un horario específico para practicar \\
IV 2 & Les enseño a mis estudiantes a establecer objetivos precisos a largo plazo \\
IV 3 & $\begin{array}{l}\text { Les enseño a mis estudiantes a establecer objetivos precisos a largo y mediano } \\
\text { plazo }\end{array}$
\end{tabular}

IV 4 Les enseño a mis estudiantes a establecer objetivos precisos a largo, mediano y corto plazo

IV 5 Les pido a mis estudiantes que utilicen un diario o bitácora para anotar dudas, preguntas u observaciones y después consultarlas conmigo

IV 6 Les pido a mis estudiantes que especifiquen una cantidad de tiempo para llevar a cabo cada tarea u objetivo a lograr.

IV 7 Les pido a mis estudiantes que mantengan control sobre el tiempo dedicado a la práctica utilizando un diario, registro o tabla

IV 8 Les enseño a mis estudiantes a escribir las digitaciones en la partitura

IV 9 Les enseño a mis estudiantes a aislar los pasajes difíciles para practicarlos por separado

IV 10 Les enseño a mis estudiantes a planificar la interpretación musical antes de practicar tomando decisiones sobre carácter, estilo, tempo, etc.

IV 11 Les enseño a mis estudiantes a Analizar la partitura desde el punto de vista armónico identificando progresiones, modulaciones, inflexiones etc.

IV 12 Les enseño a mis estudiantes a analizar la estructura formal de la partitura identificando repeticiones, secciones diferentes, etc.

IV 13 Les pido a mis estudiantes que hagan anotaciones en la partitura sobre los aspectos que quiero mejorar o corregir.

IV 27 Les enseño a mis estudiantes a diseñar estrategias para vencer las dificultades técnicas o musicales de mis obras

\subsection{Dimensión de control de ejecución}

Los ítems IV-14 al IV-26 tuvieron como objetivo conocer el nivel de enseñanza de estrategias para que los estudiantes lleven a cabo la práctica efectiva de sus obras. El alfa de Cronbach para esta dimensión fue de 0.701 . 
Tabla 6. Lista de ítems que conformaron la dimensión de "control de ejecución".

\begin{tabular}{|c|c|}
\hline & Ítems \\
\hline IV 14 & Les enseño a mis estudiantes a practicar una sección a la vez \\
\hline IV 15 & Les enseño a mis estudiantes a practicar sus piezas de principio a fin sin parar \\
\hline IV 16 & $\begin{array}{l}\text { Les enseño a mis estudiantes a practicar de manera piramidal, es decir aprender } \\
\text { una sección o frase por vez e ir añadiendo secciones hasta aprender un todo }\end{array}$ \\
\hline IV 17 & $\begin{array}{l}\text { Les enseño a mis estudiantes a practicar la última frase, luego la penúltima, } \\
\text { luego la antepenúltima, etc., hasta aprender una sección completa }\end{array}$ \\
\hline IV 18 & $\begin{array}{l}\text { Les enseño a mis estudiantes a practicar partiendo de una sección en el centro e } \\
\text { ir añadiendo frases a los lados hasta completar la pieza }\end{array}$ \\
\hline IV 19 & Les enseño a mis estudiantes a practicar lentamente \\
\hline IV 20 & $\begin{array}{l}\text { Les enseño a mis estudiantes a practicar los pasajes rápidos con distintos ritmos } \\
\text { para asegurar la precisión rítmica y la sincronía. }\end{array}$ \\
\hline IV 21 & $\begin{array}{l}\text { Les enseño a mis estudiantes a practicar exagerando las articulaciones para ga- } \\
\text { rantizar precisión y sincronía }\end{array}$ \\
\hline IV 22 & Les enseño a mis estudiantes a practicar con metrónomo \\
\hline IV 23 & $\begin{array}{l}\text { Les enseño a mis estudiantes a practicar los pasajes con metrónomo y gradual- } \\
\text { mente incrementar la velocidad. }\end{array}$ \\
\hline IV 24 & $\begin{array}{l}\text { Les enseño a mis estudiantes a crear mapas mentales para entender la obra en } \\
\text { detalle }\end{array}$ \\
\hline IV 25 & $\begin{array}{l}\text { Les enseño a mis estudiantes a practicar los pasajes mentalmente creando en } \\
\text { su mente una interpretación ideal y en detalle y luego les pido que toquen esos } \\
\text { pasajes en su instrumento. }\end{array}$ \\
\hline IV 26 & $\begin{array}{l}\text { Les enseño a mis estudiantes a practicar sin su instrumento simulando los mo- } \\
\text { vimientos que haría al practicar un pasaje y luego les pido lo toquen en su } \\
\text { instrumento }\end{array}$ \\
\hline
\end{tabular}

\subsection{Dimensión de auto re-direccionamiento}

El auto re-direccionamiento implica la autoevaluación de los resultados de la práctica y la toma de decisiones para reiniciar el ciclo. Esta dimensión es esencial, si el estudiante desea obtener logros entre lección y lección y tener un avance sostenido (Boucher, Dube y Creech, 2017).

Los ítems IV-28 al IV-30 pertenecen a la dimensión de auto re-direccionamiento, y tienen como objetivo conocer el nivel de enseñanza de estrategias para evaluar y modificar la práctica. El alfa de Cronbach para esta dimensión fue de 0.754 . De acuerdo con Zimmerman (1989) la auto-evaluación implica comparar sistemáticamente el desempeño personal con un estándar o meta y se puede llevar a cabo sobre la base de criterios temporales o absolutos, conocimiento de los estándares y establecimiento de objetivos. 
Por supuesto, se supone que el estudiante debería ejercer una constante auto-evaluación de su práctica sobre la base de su criterio y del conocimiento de los estándares. Sin embargo, no siempre es así. Las investigaciones coinciden en que la auto-evaluación sin un medio de contraste o comparación suele no reflejar la realidad total (Daniel, 2001; Boucher et ál., 2017; Bugos y High, 2009; Silveira y Gavin, 2015). Por el contrario, la revisión bibliográfica indica que la auto grabación en video y en audio, así como la asesoría y evaluación de pares representan estrategias de evaluación de alto nivel metacognitivo.

Por ejemplo, Daniel (2001) llevó a cabo un estudio relacionado con la autoevaluación y encontró que muchos estudiantes se dan cuenta de muchos errores hasta después que se audio-graban. En otras palabras, no eran conscientes de esos errores durante su práctica. En ese mismo sentido, Silveira y Gavin (2015) en un estudio con 112 estudiantes de secundaria, encontraron que la percepción de estos, cambiaba en tres autoevaluaciones consecutivas y se hacía más estricta al escuchar una grabación en audio de su propia interpretación. Por lo anterior, el investigador decidió incluir solo aquellas estrategias que confrontaran directamente a los estudiantes con su propia ejecución.

Tabla 7. Ítems para medir la dimensión de "auto re-direccionamiento".

\begin{tabular}{ll}
\hline & Ítems \\
\hline IV 28 & $\begin{array}{l}\text { Les pido a mis estudiantes que video-graben su ejecución y que evalúen sus } \\
\text { resultados }\end{array}$ \\
IV 29 & $\begin{array}{l}\text { Les pido a los estudiantes que audio-graben su ejecución y que evalúen sus } \\
\text { resultados }\end{array}$ \\
IV 30 & $\begin{array}{l}\text { Les pido a mis estudiantes que inviten a sus amigos o compañeros que los } \\
\text { escuchen y les den retroalimentación sobre su ejecución }\end{array}$ \\
\hline
\end{tabular}

\subsection{Resumen estadístico del uso de estrategias}

Un resumen estadístico de las respuestas de los profesores a los treinta ítems anteriores dio los siguientes resultados:

Tabla 8. Resumen estadístico de la variable práctica efectiva.

\begin{tabular}{lll}
\hline Variable y dimensiones & Media & Categoría \\
\hline Variable práctica efectiva en general & 3.97 & Entre "algunas veces" y "frecuentemente" \\
Dimensión de preparación & 4.17 & Entre "frecuentemente" y "siempre" \\
Dimensión de control de ejecución & 3.83 & Entre "algunas veces"y "frecuentemente" \\
Dimensión de re-direccionamiento & 3.65 & Entre "algunas veces" y "frecuentemente" \\
\hline
\end{tabular}

Del resumen anterior, destaca el hecho de que la dimensión de preparación de la práctica es la única que se encuentra entre las categorías de frecuentemente y siempre. Sin embargo, es preocupante que las dimensiones de control de ejecución $y$, sobre todo, la dimensión de auto re-direccionamiento, se encuentre en un nivel medio, pues el "círculo virtuoso" no puede iniciarse de manera efectiva si no existe 
una buena autoevaluación. Lo anterior da como resultado que la variable práctica efectiva se encuentre entre "algunas veces y frecuentemente" sin poder llegar a ideal que sería la categoría "siempre".

Un estudio simultáneo a éste, llevado a cabo por el investigador en las mismas universidades, para medir el nivel de utilización de estrategias de práctica efectiva por parte de 252 estudiantes, reveló diferencias importantes entre lo que los profesores afirman pedir o enseñar a sus estudiantes y lo que éstos hacen. En ese estudio se utilizó la misma escala de 30 ítems. Cada uno de los ítems para los profesores apareció también en la encuesta para estudiantes. Por ejemplo, el ítem "Le pido a mis estudiantes que establezcan un horario específico para practicar" aparece en la encuesta para estudiantes como "Establezco un horario específico para practicar". El resumen estadístico por dimensión arrojó los siguientes resultados.

Tabla 9. Diferencia entre lo que los profesores afirman enseñar y lo que los estudiantes afirman hacer.

\begin{tabular}{|c|c|c|c|c|}
\hline \multirow{2}{*}{$\begin{array}{l}\text { Dimensión } \\
\text { - Enseñanza/implementación de es- } \\
\text { trategias correspondientes a la } \\
\text { dimensión de preparación de la } \\
\text { práctica }\end{array}$} & \multicolumn{2}{|c|}{$\begin{array}{l}\text { Media de los profesores que } \\
\text { las enseñan }\end{array}$} & \multicolumn{2}{|c|}{$\begin{array}{l}\text { Media de los estudiantes que } \\
\text { las implementan }\end{array}$} \\
\hline & 4.17 & $\begin{array}{l}\text { Entre "Frecuente- } \\
\text { mente" y "Siem- } \\
\text { pre" }\end{array}$ & 3.55 & $\begin{array}{l}\text { "Entre Algunas ve- } \\
\text { ces" y "Frecuente- } \\
\text { mente" }\end{array}$ \\
\hline $\begin{array}{l}\text { - Enseñanza/implementación de es- } \\
\text { trategias correspondientes a la } \\
\text { dimensión de control de ejecución }\end{array}$ & 3.83 & $\begin{array}{l}\text { Entre "Algunas ve- } \\
\text { ces" y "Frecuente- } \\
\text { mente" }\end{array}$ & 3.33 & $\begin{array}{l}\text { Entre "Algunas ve- } \\
\text { ces" y "Frecuente- } \\
\text { mente" }\end{array}$ \\
\hline $\begin{array}{l}\text { Enseñanza/implementación de es- } \\
\text { trategias correspondientes a la } \\
\text { dimensión de auto re-direcciona- } \\
\text { miento }\end{array}$ & 3.65 & $\begin{array}{l}\text { Entre "Algunas ve- } \\
\text { ces" y "Frecuente- } \\
\text { mente" }\end{array}$ & 2.96 & $\begin{array}{l}\text { Entre "Rara vez" y } \\
\text { "Algunas veces" }\end{array}$ \\
\hline
\end{tabular}

El nivel de significancia es de p. 0.059. Tomando en cuenta que la muestra tendría un $7 \%$ de margen de error y un $93 \%$ de nivel de confianza, los resultados son significativos pues p. $0.059<0.07$.

Así, la información anterior corrobora la idea de que no es suficiente constatar al final de la lección de instrumento que los estudiantes saben cómo practicar, sino que es necesario monitorizar la práctica de los estudiantes a fin de exhortarlos a utilizar de manera consistente estrategias de práctica efectiva y evitar, en la medida de lo posible, que se extravíen y pierdan el tiempo practicando de manera incorrecta.

Las preguntas números 5 y 6 " ¿Está usted satisfecho con los resultados obtenidos por sus estudiantes en sus lecciones?” y “¿Está usted satisfecho con los resultados obtenidos por sus estudiantes en sus presentaciones?" recibieron respuestas muy similares. En la gráfica pueden percibirse altos porcentajes en la categoría "frecuentemente" y una diferencia muy importante para la categoría "siempre". Ante los resultados, surgen los cuestionamientos ¿A qué se debe que la categoría "siempre" haya obtenido un porcentaje tan bajo? ¿Qué factor estará ocasionando que la categoría "siempre" no obtenga un mayor porcentaje? 


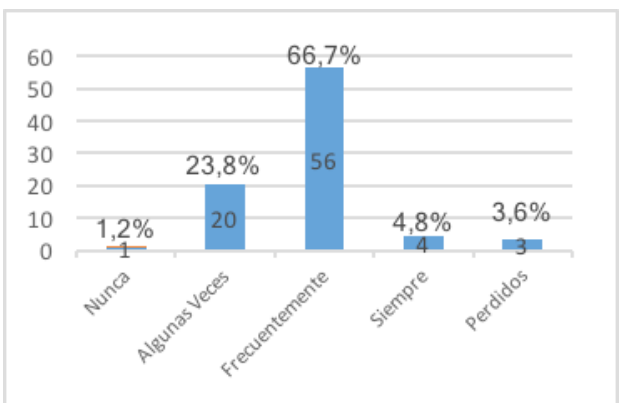

Figura 3. ¿Está Usted satisfecho con los resultados obtenidos por sus estudiantes en sus lecciones de instrumento?

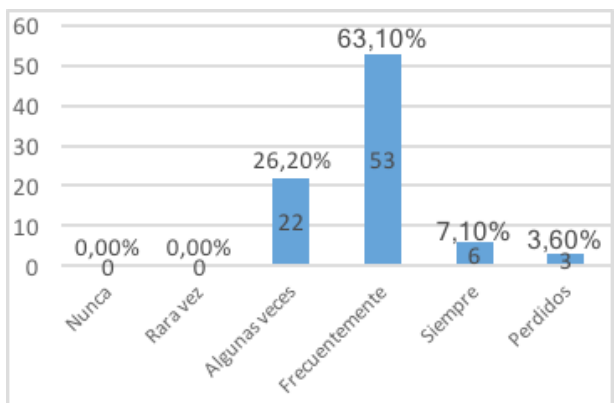

Figura 4. ¿Está Usted satisfecho con los resultados obtenidos por sus estudiantes en sus presentaciones?

Al llevar a cabo varias correlaciones estadísticas se encontró, con un coeficiente bajo, pero positivo de $r=0.325$, una correlación entre la variable "nivel de satisfacción de los profesores con los resultados obtenidos en sus presentaciones" y el nivel de uso de estrategias de práctica efectiva en la dimensión de "control de ejecución". Esta correlación estadística sugiere que, al aumentar el uso de estrategias de control de ejecución aumenta también el nivel de satisfacción de los profesores con las presentaciones de sus estudiantes.

Tabla 10. Correlación entre la satisfacción con las presentaciones y la dimensión de control de ejecución.

\begin{tabular}{|c|c|c|c|}
\hline \multirow{4}{*}{$\begin{array}{l}\text { ¿Está usted satisfecho con } \\
\text { los resultados obtenidos } \\
\text { por sus estudiantes en sus } \\
\text { presentaciones (exáme- } \\
\text { nes, recitales, audiciones, } \\
\text { etc.? }\end{array}$} & & $\begin{array}{l}\text { ¿Está usted satisfecho } \\
\text { con los resultados obte- } \\
\text { nidos por sus estudiantes } \\
\text { en sus presentaciones }\end{array}$ & $\begin{array}{l}\text { Control de la } \\
\text { Ejecución }\end{array}$ \\
\hline & $\begin{array}{l}\text { Correlación de } \\
\text { Pearson }\end{array}$ & 1 & .325 \\
\hline & Sig. (2 colas) & & 0.01 \\
\hline & $\mathrm{N}$ & 81 & 62 \\
\hline \multirow[t]{3}{*}{ Control de la Ejecución } & $\begin{array}{l}\text { Correlación de } \\
\text { Pearson }\end{array}$ & .325 & 1 \\
\hline & $\begin{array}{l}\text { Significancia. (2 } \\
\text { colas) }\end{array}$ & 0.01 & \\
\hline & $\mathrm{N}$ & 62 & 62 \\
\hline
\end{tabular}

Para la pregunta 7, "¿Qué tipo de recursos podrían contribuir para que los estudiantes mejoren su desempeño como ejecutantes?" se proporcionaron las siguientes opciones:

1. La inclusión de un curso sobre pedagogía del instrumento.

2. La impartición de más clases magistrales. 
3. Mayor supervisión por parte de los profesores sobre la manera como practican los estudiantes.

4. Mayor concientización por parte de los estudiantes sobre la importancia de utilizar estrategias de práctica adecuadas.

5. Otros.

En esta pregunta, los profesores podían seleccionar las opciones que consideraran necesarias para lograr un mejor desempeño de sus estudiantes. A continuación, se presenta una tabla que muestra las frecuencias y porcentajes de sus respuestas.

Tabla 11. Respuestas de los profesores sobre los recursos que podrían contribuir para que los estudiantes mejoren su desempeño como ejecutantes.

\begin{tabular}{lll}
\hline Categoría & Frecuencia & Porcentaje \\
$\begin{array}{l}\text { La inclusión de un curso sobre pedagogía } \\
\text { del instrumento }\end{array}$ & 24 & $28.57 \%$ \\
$\begin{array}{l}\text { La impartición de más clases magistrales } \\
\text { Mayor supervisión por parte de los profe- }\end{array}$ & 26 & $30.95 \%$ \\
$\begin{array}{l}\text { sores en la manera como practican los es- } \\
\text { tudiantes }\end{array}$ & $36.90 \%$ \\
$\begin{array}{l}\text { Mayor concientización por parte de los es- } \\
\text { tudiantes sobre la importancia de utilizar }\end{array}$ & 51 & $60.71 \%$ \\
estrategias de práctica adecuadas & 14 & \\
Otro & & \\
\hline
\end{tabular}

La opción "mayor concientización por parte de los estudiantes sobre la importancia de utilizar estrategias de práctica adecuadas" obtuvo el mayor número de frecuencias. Sin embargo, dada la importancia de la práctica efectiva en la formación de un ejecutante, se esperaría un porcentaje mucho más alto.

Un $36.90 \%$ de los profesores seleccionó la opción "mayor supervisión por parte de los profesores en la manera como practican los estudiantes", porcentaje que sugiere poca preocupación por parte del profesorado sobre lo que debería ser parte de su labor docente (Gaunt, 2008; Jorgensen, 2000).

Con una frecuencia de 26 y un porcentaje de $30.95 \%$ aparece la opción b: "la impartición de más clases magistrales". Esta respuesta se conecta con la pregunta número 9: “¿Cómo enseña en sus lecciones de instrumento?” en la que $29.09 \%$ de los profesores afirmaron enseñar tomando ideas de clases magistrales.

La opción con menos frecuencias correspondió a "la inclusión de un curso sobre pedagogía del instrumento". En el caso del piano, existen maestrías y doctorados en pedagogía del instrumento. Del mismo modo, se ha desarrollado en muchas partes la pedagogía de diversos instrumentos con el fin de fundamentar su enseñanza con información y metodologías que coadyuven al desarrollo técnico y musical de los estudiantes de una manera armónica y sana (por ejemplo, evitando las lesiones). Esta opción solo recibió 24 frecuencias con un porcentaje de $16.44 \%$ de profesores.

La pregunta ocho buscaba conocer la frecuencia con que se impartían cursos de formación docente a los profesores de instrumento. Los cursos de formación docente tienen como objetivo actualizar y preparar al profesor para una mejor impartición de 
su materia, lo cual redunda en un mejoramiento en el desempeño del estudiante. El beneficio de los cursos de formación docente se incrementa si se imparten de manera continua y sistemática (Harwell, 2003). La pregunta “¿Qué tan frecuentemente se imparten cursos sobre pedagogía del instrumento en su escuela, que aborden el tema de las estrategias de práctica?" obtuvo respuestas precarias que sugieren poco interés por parte de las instituciones para mantener en constante formación a su profesorado.

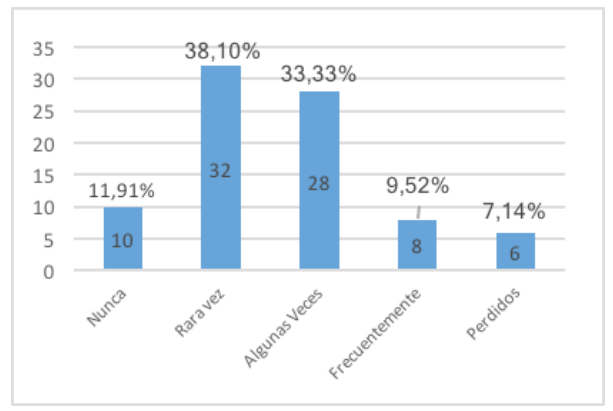

Figura 5. Frecuencia con que se imparten cursos sobre pedagogía del instrumento en las escuelas participantes en donde se aborde el tema de las estrategias de práctica.

El ítem nueve “¿Cómo enseña en sus lecciones de instrumento?” incluyó las siguientes cinco opciones de respuesta:

1. Enseño como me enseñaron mis profesores.

2. Tomo ideas de mis profesores y de profesores que he visto en clases magistrales.

3. Desarrollo mis propias ideas sobre cómo enseñar.

4. Recurro a resultados de investigación publicados en revistas especializadas.

5. He tomado cursos de pedagogía del instrumento.

Las respuestas más seleccionadas fueron "tomo ideas de mis profesores y de profesores que he visto en clases magistrales", con un 29.09\% y "Desarrollo mis propias ideas sobre cómo enseñar" igual con un 29.09\%. Ambas preferencias, aunque buenas, son limitadas. La primera porque se reduce al número de profesores que se haya tenido, así como al número de profesores que haya visto impartiendo clases magistrales. La segunda está limitada a la propia creatividad e inquietud del profesor. La opción "He tomado cursos de pedagogía del instrumento" ocupó el tercer lugar de preferencia con un $17.73 \%$. La opción "recurro a resultados de investigación publicados en revistas especializadas", ocupa un preocupante cuarto lugar con un $13.18 \%$ de preferencia por parte de los profesores. En este respecto, los resultados de la encuesta sugieren que mucha información, resultado de estudios empíricos sobre estrategias de práctica efectiva, no es aprovechada en beneficio de los estudiantes y del propio profesor. Finalmente, la opción "Enseño como me enseñaron mis profesores" ocupó el último lugar con un $10.91 \%$ de preferencia. Aun cuando el panorama general sobre cómo enseñan los profesores no es el más prometedor, la baja incidencia en esta respuesta sugiere que al menos, la mayoría no se limita a enseñar de una sola manera, sino que busca otras opciones pedagógicas. 


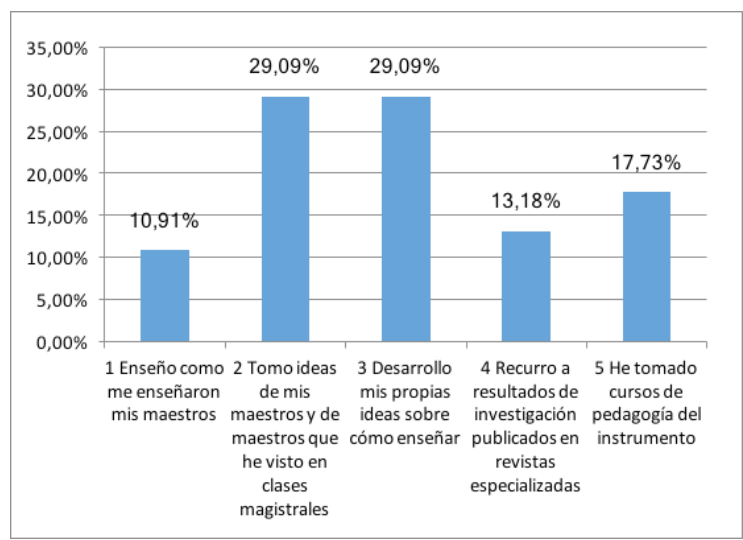

Figura 6. Manera como enseñan los profesores.

\section{Conclusiones}

Respecto al primer objetivo de este estudio "Identificar las estrategias de práctica efectiva y autorregulación que los maestros enseñan a sus estudiantes cuando éstos se preparan para una actuación y la medida en que cubren las dimensiones de preparación, control de ejecución y auto re-direccionamiento definidas por Zimmerman $(1998,2000) "$, los resultados sugieren que los profesores encuestados estaban comprometidos con su labor docente y que enseñaban estrategias de práctica efectiva a sus estudiantes cubriendo las tres dimensiones señaladas. Sin embargo, el análisis estadístico descriptivo reveló que, aun cuando la dimensión de preparación de la práctica se encontraba en un nivel alto, las dimensiones de control de ejecución y de auto re-direccionamiento se encontraban en niveles medios, lo que se presentaba como un área para mejorar. Los principales aspectos que podían ser fortalecidos en la primera dimensión, estuvieron representados principalmente por la falta de utilización de estrategias que promovieran niveles altos de metacognición. Respecto a la segunda dimensión, hacía falta un mayor uso/enseñanza de estrategias eficaces para evaluar la práctica, tales como la auto grabación en audio y la auto grabación en video. Este último aspecto tiene una importancia vital, ya que una fase consolidada de auto re-direccionamiento impulsa el reinicio del ciclo auto-regulatorio de una manera más efectiva, incrementa la motivación y promueve el crecimiento musical.

En cuanto a los objetivos dos y tres ("determinar el grado en que los maestros monitorizan la práctica de sus estudiantes" y "determinar la manera como los maestros monitorizan la práctica de sus estudiantes"), los resultados reflejaron algunas áreas de mejora pues, aun cuando un $65.5 \%$ de los profesores manifestó que monitorizaba la práctica "siempre" y "frecuentemente", sólo un $21 \%$ de ellos verificaba la manera como los estudiantes lo hacían. Como se discutió anteriormente, los estudiantes (sobre todo los más inexpertos) podrían estar practicando de una manera 
poco productiva, por lo que es muy importante monitorizar su práctica y, a través de un proceso de análisis y reflexión entre el alumno y el maestro, seleccionar y/o crear estrategias de práctica efectiva que contribuyan al logro de resultados musicales sólidos y confiables.

En relación al objetivo cuatro ("determinar el nivel de satisfacción de los maestros respecto a los resultados que los estudiantes obtienen en sus sesiones de práctica y en sus presentaciones"), destacó el hecho de que un alto porcentaje de los profesores, tuviera un nivel muy alto de satisfacción en la categoría de "frecuentemente", mientras que el índice bajaba dramáticamente en la categoría de "siempre". Como se vio anteriormente, el análisis estadístico descriptivo reveló que la dimensión de control de ejecución se encontraba en un nivel medio. Al llevar a cabo una correlación estadística se obtuvo un coeficiente $r$ de Pearson significativo, aunque bajo, entre la dimensión de control de ejecución y el nivel de satisfacción, lo que podría explicar el bajo índice en la categoría "siempre". En otras palabras, un mayor énfasis en la enseñanza de estrategias en la dimensión de control de ejecución, sobre todo de aquellas que promueven niveles metacognitivos altos, redundaría en un mejor desempeño de los estudiantes y elevaría el nivel de satisfacción de los profesores en la categoría de siempre.

El objetivo seis consistió en conocer la opinión de los maestros respecto al tipo de recursos que podrían contribuir para que los estudiantes mejoraran su desempeño como ejecutantes. De las respuestas, destacó que un $60.71 \%$ de los profesores consideraba importante el que hubiera una mayor concientización por parte de los estudiantes sobre la importancia de utilizar estrategias de práctica adecuada, mientras que un $36.90 \%$ consideraba que debía haber una mayor supervisión por parte de los profesores en la manera como practicaban los estudiantes. Las respuestas se relacionan con el propósito principal de este artículo. Sin embargo, dada la importancia de la práctica efectiva en la formación del estudiante se esperaría porcentajes mucho más altos en estas opciones de respuesta.

Los aspectos más neurálgicos revelados en este estudio, están representados por los resultados correspondientes a los objetivos 7 ("determinar la frecuencia con que los maestros reciben cursos sobre pedagogía del instrumento que aborden el tema de las estrategias de práctica") y 8 ("caracterizar la filosofía de los maestros en cuanto a la enseñanza instrumental"). Por un lado, el estudio reveló una participación muy baja por parte de los profesores en cursos de formación docente. Por otro lado, solo un $13.18 \%$ de los profesores apoyaba su práctica en resultados de investigación. Ambos factores se conjugan, pues usualmente, la formación docente continua recurre a los resultados de investigación con propósito de innovación y para promover una mejora de los procesos de enseñanza y aprendizaje. Definitivamente, se trata de dos aspectos de la docencia instrumental que deben ser atendidos a nivel institucional y que redundarán en beneficio de maestros y estudiantes.

De la información contextual obtenida, merece mención especial el nivel académico de los profesores encuestados, ya que un alto porcentaje de ellos contaba por lo menos con una licenciatura $(88.1 \%)$. Este es un dato importante y alentador. La primera licenciatura en música con reconocimiento de validez oficial se ofreció en México hasta 1968 (UNAM, S.F.). Debieron pasar 41 años para que estados como Aguascalientes y Nayarit pudieran ofrecer una licenciatura en música. Así, por muchos años, muchas instituciones ofrecieron licenciaturas en música a pesar de no contar con profesores con ese nivel académico. La información obtenida indica un 
aumento importante en el número de profesionales de la música en México que ahora atiende las necesidades de formación musical en instituciones del país. Del mismo modo, el número de profesores con posgrado ha aumentado, lo cual, como se vio en el estudio, está teniendo un impacto en la formación de los estudiantes. Dado el impulso y apoyo que el Consejo Nacional de Ciencia y Tecnología (CONACYT) ha estado otorgando a la formación de profesionales con posgrados, es probable que en algunos años se incremente su número.

Finalmente, la información obtenida a través de este estudio, podría constituirse en el fundamento y justificación para impulsar una mejora en la preparación de los profesores y estudiantes a través de cursos, talleres y conferencias que aborden el aspecto de la enseñanza, implementación y monitorización de estrategias de práctica efectiva. También podría impulsar la inclusión de un curso en el plan de estudios, en el que se aborden los aspectos fundamentales de la práctica instrumental y se proporcione a los estudiantes los conocimientos básicos sobre estrategias de práctica efectiva necesarias para trabajar sus piezas de manera más productiva y saludable.

En general, podría decirse que existen aspectos mejorables importantes en el ámbito de la formación de músicos ejecutantes en la Región Centro Occidente de la ANUIES en México. Sin embargo, esos aspectos podrían ser fácilmente subsanados, dada la actitud positiva y la gran disposición que mostraron los profesores encuestados durante el estudio y que dejó ver en todo momento su interés en la mejora docente. Sirva la información consignada en este artículo para contribuir en la promoción de acciones que redunden en una mejor preparación de los profesores y en una formación más sólida de los estudiantes.

\section{Referencias Bibliográficas}

Barry, N. (1990). The effects of practice strategies, individual differences in cognitive styles, and sex upon technical accuracy and musicality of student instrumental performance (Tesis Doctoral). Recuperado de ProQuest Dissertations Publishing. (Orden 9100056)

Barry, N. y McArthur, V. (1994). Teaching strategies in the music studio: A survey of applied music teachers. Psychology of Music, 22, 44-45. doi: 10.1177/0305735694221004

Bernstein, S. (1981). With your own two hands. New York: G. Schirmer, Inc.

Berr, B. (2010). The end is where we start from. American Music Teacher, June-July, 56. Recuperado de http://web.a.ebscohost.com.dibpxy.uaa.mx/ehost/pdfviewer/pdfviewer?vid=4\&sid=9f22f677-c469-4675-a33b-8033bbbfebc0\%40sessionmgr4010\&hid=4206

Boucher, M., Dube, F. y Creech, A. (2017). The effect of video feedback on the self-assessment of a music performance by college level classical guitarists. In G. Hughes (Ed.), Ipsative assessment and learning gains: Case studies from international practitioners. (pp. 197-220). Basingstoke, Hampshire: Palgrave Macmillan.

Brooks, R. W. (1995). Mental practice and the musician. A practical approach to practice. Update: Applications of Research in Music Education, 13(2), 4-8. doi: $10.1177 / 875512339501300202$

Bugos, J. A, y High, L. (2009). Perceived versus actual practice strategy used by older adult novice piano students. Visions of Research in Music Education, 13. Recuperado de http:// www-usr.rider.edu/ vrme/

Byo, J. y Cassidy, J. W. (2008). An exploratory study of time use in the practice of music 
majors self-report and observation analysis. Update: Applications of Research in Music Education, 27(1), 33-40. doi: 10.1177/8755123308322272

Coffman, D. D. (1990). Effects of mental practice, physical practice, and knowledge of results on piano performance. Journal of Research in Music Education, 38, 187-196. doi: $10.2307 / 3345182$

Cremaschi, A. (2012). The effect of a practice checklist on practice strategies, practice self-regulation and achievement of collegiate music majors enrolled in a beginning class piano course. Research Studies in Music Education, 34(2), 223-233. doi: $10.1177 / 1321103$ X12464743

Creswell, J. W. (2001). Educational Research: Planning, Conducting, and Evaluating Quantitative and Qualitative Research. Boston, MA: Pearson.

Daniel, R. (2001). Self-assessment in performance. British Journal of Music Education. 18(3), 215-226. doi: https://doi.org/10.1017/S0265051701000316

Duke, R. A., Simmons, A. L. y Davis Cash, C. (2009). It's not how much; it's how. Characteristics of practice behaviour and retention of performance skills. Journal of Research in Music Education, 56(4), 310-321. doi: 10.1177/0022429408328851

Flavell, J.H., Beach, D.R. y Chinsky, J.M. (1966). Spontaneous verbal rehearsal in a memory task as a function of age. Child Development, 37, 283-299. doi: 10.2307/1126804

Gaunt, H. (2008). One-to-one tuition in a conservatoire: the perceptions of instrumental and voice teachers. Psychology of Music, 36, 215-245. doi: 10.1177/0305735607080827

Gregg, M. Clark, T. y Hall, C. (2008). Seeing the sound: An exploration of the use of mental imagery by classical musicians. Musicae Scientiae, 12(2), 231-247. doi: $10.1177 / 102986490801200203$

Gregg, M. y Clark, T. (2007). Theoretical and practical applications of mental imagery. International Symposium on Performance Science, 295-300. Recuperado de http://www. performancescience.org/ISPS2007/Proceedings/Rows/51Gregg\%20etal.pdf

Hallam, S. (1995). Professional musician's approaches to the learning and interpretation of music. Psychology of Music, 23, 111-128. Doi: 10.1177/0305735695232001

Hallam, S. (2001a). The development of meta-cognition in musicians: Implications for education. The British Journal of Music Education, 18(1), 27-39. doi: https://doi.org/10.1017/ S0265051701000122

Hallam, S. (2001b). The development of expertise in young musicians: Strategy use, knowledge acquisition and individual diversity. Music Education Research, 3(1), 7-23. doi. org/10.1080/1461380002002991

Hallam, S., Rinta T., Varvarigou, M., Creech, A. Papageorgi, L., Gomes, T. y Lanipekun, J. (2012). The development of practising strategies in young people. Psychology of Music, 40(5), 652-680. doi: 10.1177/0305735612443868

Harwell, Sandra. (2003). Teacher Professional Development: It's Not an Event, It's a Process, CORD. Recuperado de http://www.cord.org/uploadedfiles/HarwellPaper.pdf

Herrera, M. y Cremades, R. (2014). Memorisation in piano students: A study in the Mexican context. Musicae Scientiae, 2014, 18(2), 216-231. doi. 10.1177/1029864914527105

Holmes, P. (2005). Imagination in practice: A study of the integrated roles of interpretation, imagery and technique in the learning and memorisation processes of two experienced solo performers. British Journal of Music Education, 22(03), 217 - 235. doi: 10.1017/ S0265051705006613

Johnson, R. (2011). Musical tempo stability in mental practice: A comparison of motor and non-motor imagery techniques. Research Studies in Music Education, 33(1) 3-30. doi: $10.1177 / 1321103 \times 11400501$ 
Jorgensen, H. (2000). Student learning in higher instrumental education: who is responsible? British Journal of Music Education, 17(01), 67-77.

Kim, S. J. (2008). Using a practice diary to promote self-regulated instrumental practice. American String Teacher, 58(3), 34-36. Recuperado de https://www.astastrings.org/ App_Themes/Public/Uploads/AST\%20Past\%20Issues/Aug2008_journal.pdf

Koopman, C., Smith, N., de Vugt, A., Deneer, P. y den Ouden, J. (2007). Focus on practice-relationships between lessons on the primary instrument and individual practice in conservatoire education. Music Education Research, 9(3), 373-397. Recuperado de http:// dx.doi.org/10.1080/14613800701587738

Kostka, M. J. (2002). Practice expectations and attitudes: A survey of college-level music teachers and students. Journal of Research in Music Education, 50(2), 145-154. doi: $10.2307 / 3345818$

Lathman, G.P. (2004). The motivational benefits of goal-setting. Academy of Management Executive, 18(4), 126-129. Recuperado de http://www-2.rotman.utoronto.ca/facbios/ file/15\%20-\%20Latham\%20AME\%202004.pdf

Laukka, P. (2004). Instrumental music teachers' views on expressivity: a report from music conservatoires. Music Education Research, 6(1), 45-56. Recuperado de http://dx.doi. org/10.1080/1461380032000182821

Maynard, L. (2006). The role of repetition in the practice sessions of artist teachers and their students. Bulletin of the Council for Research in Music Education, 167, 61-72. Recuperado de http://www.jstor.org/stable/40319290

McPherson, G. E. y Zimmerman, B. J. (2002). Self-regulation of musical learning: A social cognitive perspective. In R. Colwell \& C. Richardson (Eds.), The New Handbook of Research on Music Teaching And Learning: A Project Of The Music Educators National Conference (pp. 327-347). New York: Oxford University Press.

McPherson, G. y McCormick, J. (1999). Motivational and self-regulated learning components of musical practice. Bulletin of the Council for Research in Music Education 17(141), 98-102. Recuperado de http://www.jstor.org/stable/40318992

Miklaszewski, K. (1989). A case study of a pianist preparing a musical performance. Journal of Psychology of Music, 17(2), 95-109. doi: 10.1177/0305735689172001

Miksza, P. (2006). A review of research on practicing: Summary and synthesis of the extant research with implications for a new theoretical orientation. Bulletin of the Council for Research in Music Education, 190(3), 51-92. doi: 10.5406/bulcouresmusedu.190.0051

Miksza, P. (2007). Effective practice: An Investigation of observed practice behaviors, self-reported practice habits, and the performance achievement of high school wind players. Journal of Research in Music Education, 55(4), 359-375. doi: 10.1177/0022429408317513

Miksza, P. (2011). Relationships among achievement goal motivation, impulsivity, and the music practice of collegiate brass and woodwind players. Psychology of Music, 39(1) 50 67. doi: $10.1177 / 0305735610361996$

Nielsen, S. (1999). Learning strategies in instrumental music practice. British Journal of Music Education, 16(3): 275-291. doi: http://dx.doi.org/10.1080/14613800120089223

Nielsen, S. (2001). Self-regulating learning strategies in instrumental music practice. Music Education Research, 3(2). doi: http://dx.doi.org/10.1080/14613800120089223

Oare, S. (2012). Decisions made in the practice room: A qualitative study of middle school students' thought processes while practicing. Update: Applications of Research in Music Education, 30(2), 63-70. doi: 10.1177/8755123312437051

Pearce, E. (1992). The other teacher: Home practice. Keyboard Companion, (3), 8-9.

Reybrouck, M. (2009). Musical imagery between sensory processing and ideomotor simula- 
tion. En R. I. Godoy y H. Jorgensen (Eds.), Studies on New Musical Research: Musical Imagery (pp. 117-136). New York: Taylor and Francis.

Rosenthal, R., Durairaj, M. y Magann, J. (2009). Musicians' descriptions of their expressive musical practice. Bulletin of the Council for Research in Music Education, 181(4), 37-49.

Sheldon, D. A. (2005). Peer and cross-age tutoring in music. Journal of Research in Music Education, 53(1), 40-50. doi: 10.2307/3399690

Shockley, R. P. (1997). Mapping Music: For Faster Learning and Secure Memory - A Guide for Piano Teachers and Students. Middleton, Wisconsin: A-R Editions.

Silveira, J. y Gavin, R. (2015). The effect of audio recording and playback on self-assessment among middle school instrumental music students. Psychology of Music, doi: 1-13. $10.1177 / 0305735615596375$

Sloboda, J. A., Davidson, J. W., Howe, M. J. A. y Moore, D. G. (1996). The role of practice in the development of performing musicians. British Journal of Psychology, 87, 287-309. doi: 10.1111/j.2044-8295.1996.tb02591.x

Swanson, R. y Law, B. (1993). Whole-part-whole Learning model. Performance Improvement Quarterly, 6(1), 43-53. doi: 10.1111/j.1937-8327.1993.tb00572.x

Teixeira-Dos Santos, R y Hentschke, L. (2011). Praxis and poiesis in piano repertoire preparation. Music Education Research, 13(3), 273-292. Recuperado de http://dx.doi.org/10.1 080/14613808.2011.603042

Theiler, A. M. y Lippman, L. G. (1995). Effects of mental practice and modeling on guitar and vocal performance. The Journal of General Psychology, 122(4), 329-343.

UNAM. (S.F.). Presentación del Programa de Maestría y Doctorado 2011. Escuela Nacional de Música. Recuperado de: http://www.posgrado.unam.mx/musica/div/planEstudios $2011 /$ pdf/Presentaci $\%$ C3\%

Westney, W. (2003). The Perfect Wrong Note: Learning to Trust Your Musical Self. Cambridge: Amadeus Press.

Williamon, A. y Valentine, E. (2000). Quantity and quality of musical practice as predictors of performance quality. British Journal of Psychology, 91, 353-376. doi: 10.1348/000712600161871

Young, V., Burwell, K. y Pickup, D. (2003). Areas of study and teaching strategies in instrumental teaching: a case study research project. Music Education Research, 5(2), 139-155. doi: 10.1080/1461380032000085522

Zimmerman, B.J. (1989). A social cognitive view of self-regulated academic learning. Journal of Educational Psychology, 81(3), 329-339.

Zimmerman, B.J. (1998). Developing self-fulfilling cycles of academic regulation: an analysis of exemplary instructional models. En D.H.S Chunk y B.J. Zimmerman (Eds.). Self-regulated Learning. From Teaching to Self-reflective Practice (pp. 1-20). New York, Guilford Press.

Zimmerman, B.J. (2000) Attaining self-regulation: a social cognitive perspective. En M. Boekaerts, P.R. Pintrich y M. Zeidner (Eds.), Handbook of Self-regulation (pp. 13-35) San Diego: Academic Press.

Zimmerman, B. J. (2008). Investigating self-regulation and motivation: Historical background, methodological developments, and future prospects. American Educational Research Journal, 45(1), 166 -183. doi: 10.3102/0002831207312909 


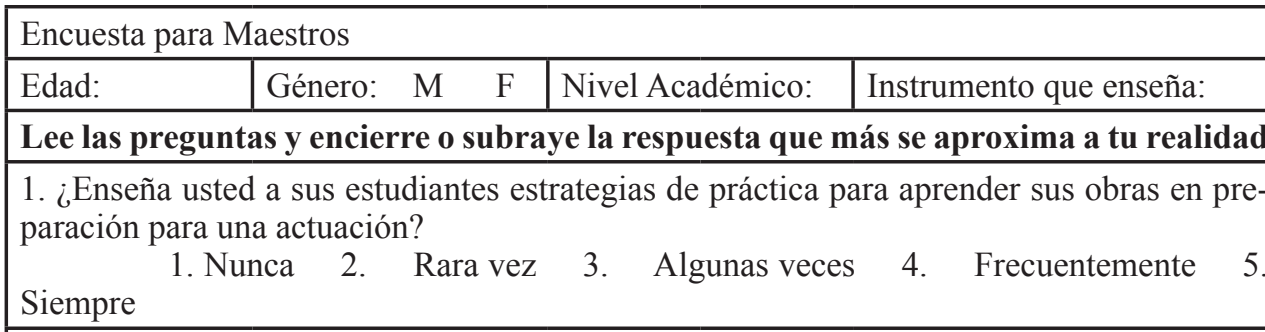

2. ¿Monitoriza usted la manera como practican sus estudiantes?

1. Nunca 2. Rara vez 3. Algunas veces 4. Frecuentemente 5. Siempre

3. ¿Cómo monitoriza usted la práctica de sus estudiantes?

a) Por los resultados que ellos obtienen durante la lección

b) Les pido que practiquen delante de mi

c) Antes de terminar la lección me cercioro de que saben cómo van a practicar

d) Considero que los estudiantes deben responsabilizarse de su propio aprendizaje. Yo solamente los oriento

c) Otro

4. Lee cuidadosamente las siguientes declaraciones y circule un número de acuerdo a las siguientes instrucciones:
1. Nunca
2. Rara vez
3. Algunas veces
4. Frecuentemente
5. Siempre

Les pido a mis alumnos que establezcan un horario específico para practicar

$$
\begin{array}{lllll}
1 & 2 & 3 & 4 & 5
\end{array}
$$

Les pido a mis alumnos que establezcan objetivos precisos a largo plazo

$$
\begin{array}{lllll}
1 & 2 & 3 & 4 & 5
\end{array}
$$

Les pido a mis alumnos que establezcan objetivos precisos a largo y mediano plazo

$$
\begin{array}{lllll}
1 & 2 & 3 & 4 & 5
\end{array}
$$

Les pido a mis alumnos que establezcan objetivos precisos a largo, mediano y corto plazo

$$
\begin{array}{lllll}
1 & 2 & 3 & 4 & 5
\end{array}
$$

Les pido a mis alumnos que utilicen un diario o bitácora para anotar dudas, preguntas u observaciones y después consultarlas conmigo

$$
\begin{array}{lllll}
1 & 2 & 3 & 4 & 5
\end{array}
$$

Les pido a mis alumnos que especifiquen una cantidad de tiempo para llevar a cabo cada tarea u objetivo a lograr.

$$
\begin{array}{lllll}
1 & 2 & 3 & 4 & 5
\end{array}
$$

Le pido a mis alumnos que mantengan control sobre el tiempo utilizando una diario, registro o tabla

$$
\begin{array}{lllll}
1 & 2 & 3 & 4 & 5
\end{array}
$$

Les enseño a mis alumnos a practicar de manera piramidal, es decir aprendiendo una sección o frase por vez y añadiendo secciones hasta que aprenden un todo.

$$
\begin{array}{lllll}
1 & 2 & 3 & 4 & 5
\end{array}
$$

Les enseño a mis alumnos a practicar la última frase, luego la penúltima, luego la antepenúltima hasta que aprenden una sección completa.

$$
\begin{array}{lllll}
1 & 2 & 3 & 4 & 5
\end{array}
$$

Les enseño a mis alumnos a practicar partiendo de una sección en el centro e ir añadiendo frases a los lados hasta completar la pieza

$$
\begin{array}{lllll}
1 & 2 & 3 & 4 & 5
\end{array}
$$

Les pido a mis alumnos que practiquen lentamente

$$
\begin{array}{lllll}
1 & 2 & 3 & 4 & 5
\end{array}
$$

Les pido a mis alumnos que practiquen los pasajes rápidos con distintos ritmos para asegurar la precisión rítmica y la sincronía.

$$
\begin{array}{lllll}
1 & 2 & 3 & 4 & 5
\end{array}
$$

Les pido a mis alumnos que practiquen exagerando las articulaciones

$$
\begin{array}{lllll}
1 & 2 & 3 & 4 & 5
\end{array}
$$


Les pido a mis alumnos que escriban las digitaciones en la partitura

$$
\begin{array}{lllll}
1 & 2 & 3 & 4 & 5
\end{array}
$$

Les enseño a mis alumnos a aislar los pasajes difíciles para practicarlos por separado

$$
\begin{array}{lllll}
1 & 2 & 3 & 4 & 5
\end{array}
$$

Les enseño a mis alumnos a planificar la interpretación musical antes de practicar tomando decisiones sobre carácter, estilo, tempo, etc.

$$
\begin{array}{lllll}
1 & 2 & 3 & 4 & 5
\end{array}
$$

Les enseño a mis alumnos a analizar la partitura desde el punto de vista armónico identificando progresiones, modulaciones, inflexiones etc.

$$
\begin{array}{lllll}
1 & 2 & 3 & 4 & 5
\end{array}
$$

Les enseño a mis alumnos a analizar la estructura formal de la partitura identificando repeticiones, secciones diferentes, etc.

$$
\begin{array}{lllll}
1 & 2 & 3 & 4 & 5
\end{array}
$$

Les pido a mis alumnos que hagan anotaciones en la partitura sobre los aspectos que quiero mejorar o corregir.

$$
\begin{array}{lllll}
1 & 2 & 3 & 4 & 5
\end{array}
$$

Les pido que practiquen una sección a la vez

$$
\begin{array}{lllll}
1 & 2 & 3 & 4 & 5
\end{array}
$$

Les pido que practiquen sus piezas de principio a fin sin parar

$$
\begin{array}{lllll}
1 & 2 & 3 & 4 & 5
\end{array}
$$

Les enseño a mis alumnos a simular los movimientos que harían al practicar un pasaje sin el instrumento y luego les pido que lo hagan con el instrumento

$$
\begin{array}{lllll}
1 & 2 & 3 & 4 & 5
\end{array}
$$

Les enseño a mis alumnos a practicar con metrónomo

$$
\begin{array}{lllll}
1 & 2 & 3 & 4 & 5
\end{array}
$$

Les pido a mis alumnos que practiquen los pasajes con metrónomo y gradualmente incrementen la velocidad.

$$
\begin{array}{lllll}
1 & 2 & 3 & 4 & 5
\end{array}
$$

Les enseño a mis alumnos a crear mapas mentales para entender la obra en detalle

$$
\begin{array}{lllll}
1 & 2 & 3 & 4 & 5
\end{array}
$$

Les enseño a mis alumnos a practicar los pasajes mentalmente creando en su mente una interpretación ideal y en detalle y luego les pido que la toquen en el instrumento.

$$
\begin{array}{lllll}
1 & 2 & 3 & 4 & 5
\end{array}
$$

Les enseño a mis alumnos a diseñar estrategias para vencer las dificultades técnicas o musicales de sus obras

$$
\begin{array}{lllll}
1 & 2 & 3 & 4 & 5
\end{array}
$$

Les pido a mis alumnos que se video-graben para evaluar los resultados

$$
\begin{array}{lllll}
1 & 2 & 3 & 4 & 5
\end{array}
$$

Les pido a mis alumnos que se audio-grabo para evaluar los resultados.

$$
\begin{array}{lllll}
1 & 2 & 3 & 4 & 5
\end{array}
$$

Les pido a mis alumnos que inviten a sus amigos o compañeros que los escuchen y les den retroalimentación sobre su ejecución

$$
\begin{array}{lllll}
1 & 2 & 3 & 4 & 5
\end{array}
$$

Dejo a mis alumnos en libertad de escoger la manera cómo deben practicar.

$$
\begin{array}{lllll}
1 & 2 & 3 & 4 & 5
\end{array}
$$

1. ¿Está usted satisfecho con los resultados obtenidos por sus estudiantes en sus lecciones de instrumento? 1 Nunca 2 Rara vez 3 Algunas veces 4 Frecuentemente 5 Siempre

2. ¿Está usted satisfecho con los resultados obtenidos por sus estudiantes en sus presentaciones (exámenes, recitales, audiciones, etc.)?

1 Nunca 2 Rara vez 3 Algunas veces 4 Frecuentemente 5 Siempre


3. ¿Qué tipo de recursos podrían contribuir para que los estudiantes mejoren su desempeño como ejecutantes?

1 La inclusión de un curso sobre pedagogía del instrumento

2 La impartición de más clases magistrales

3 Mayor supervisión por parte de los maestros en la manera como practican los estudiantes

4 Mayor concientización por parte de los estudiantes sobre la importancia de utilizar estrategias de práctica adecuadas

5 Otros:

4. ¿Qué tan frecuentemente se imparten cursos sobre pedagogía del instrumento en su escuela, que aborden el tema de las estrategias de práctica?

1 Nunca 2 Rara vez 3 Algunas veces 4 Frecuentemente 5 Siempre

5. ¿Cómo enseña en sus lecciones de instrumento? (circule las opciones que se apliquen a su caso)

1 Enseño como me enseñaron mis maestros

2 Tomo ideas de mis maestros y de maestros que he visto en clases magistrales

3 Desarrollo mis propias ideas sobre cómo enseñar

4 Recurro a resultados de investigación publicados en revistas especializadas

5 He tomado cursos de pedagogía del instrumento 